\title{
DNA-Ploidie und proliferative Aktivität bei Speicheldrüsentumoren
}

\author{
Oliver Driemel ${ }^{1}$, Klaus Kraft ${ }^{2}$ und Jörg Hemmer ${ }^{3,4}$ \\ (1) Klinik und Poliklinik für Mund-, Kiefer- und Gesichtschirurgie, Universität Regensburg, Franz-Josef-Strauß-Allee 11, \\ 93053 Regensburg, Germany \\ (2) Abteilung Pathologie, Bundeswehrkrankenhaus UIm, Oberer Eselsberg 40, 89081 Ulm, Germany \\ (3) Abteilung Pathologie, Universität Ulm, Albert-Einstein-Allee 11, $89081 \mathrm{UIm}$, Germany \\ (4) Department of Oral Pathology, University of Pretoria, 0002 Pretoria, South Africa
}

\section{Zusammenfassung}

Mithilfe hoch-auflösender DNA-Durchflusszytophotometrie wurden DNA-Ploidie und SPhasenfraktion (SPF) bei 279 Speicheldrüsentumoren bestimmt. Alle 229 benignen Neoplasien waren diploid; 12 von 50 malignen Tumoren wiesen Zellpopulationen mit aneuploidem DNA-Gehalt auf. Die SPF-Werte der diploiden Malignome waren signifikant höher als bei pleomorphen Adenomen, unterschieden sich jedoch nicht von denen der Zystadenolymphome (Warthin-Tumoren). Während Aneuploidie ein Malignitätsmerkmal darstellt, ist der SPF-Wert für die Dignitätsdiagnostik bei Speicheldrüsentumoren nur von eingeschränktem Nutzen.

\section{DNA ploidy and proliferative activity in salivary gland tumours}

\section{Abstract}

DNA ploidy and S-Phase fraction (SPF) of 279 salivary gland tumours were analysed using high-resolution DNA flow cytometry. All 229 benign neoplasms were diploid while 12 of 50 malignant tumours showed cell populations with aneuploid DNA content. The SPF values of diploid malignancies were significantly higher if compared with pleomorphic adenomas but did not differ from that of the zystadenolymphoma (Warthin tumour) group. While aneuploidy represents a distinct indicator of malignancy SPF values are of minor relevance for dignity assessment in salivary gland tumours.

(Vortrag gehalten bei der 36. Jahrestagung des Deutsch-Österreichisch-Schweizerischen Arbeitskreises für Tumoren im Kiefer- und Gesichtsbereich (DÖSAK) innerhalb der Deutschen Gesellschaft für Mund-, Kiefer- und Gesichtschirurgie in Bochum, 14.-15.November 2005.) 


\section{Einleitung}

Klonale Veränderungen der regulären Chromosomenzahl sind ein charakteristisches, wenn auch nicht obligates Malignitätsmerkmal solider Tumoren [8, 41]. Verlaufsstudien belegen, dass auch die gelegentliche Diagnose aneuploider Zellpopulationen in als gutartig klassifizierten Neoplasien regelmäßig als frühes Zeichen einer malignen Progression gewertet werden muss [31, 44]. Die Bestimmung der DNA-Ploidie könnte daher auch für Tumoren der Speicheldrüsen, bei denen die Abgrenzung benigner und maligner Neoplasien häufig mit Schwierigkeiten verbunden ist [42], zu einer Verbesserung der Dignitätsbewertung beitragen.

Eine einfache, schnelle und kostengünstige Technik zum Nachweis von Zellen mit umfassenden Karyotypanomalien stellt die DNA-Durchflusszytophotometrie dar [25, 35]. Mit dieser Methode konnte in umfangreichen Studien an oralen Plattenepithelkarzinomen eine Aneuploidierate von ungefähr $80 \%$ festgestellt werden, wobei sich der Ploidie-Status zudem als unabhängiger prognostischer Faktor für Überleben [18, 24], regionale Metastasierung [1922] und lokale Rezidivierung [20] erwies. Die bislang an Speicheldrüsentumoren durchgeführten Untersuchungen sind weit weniger aufschlussreich, was im Wesentlichen auf zwei Ursachen zurückgeführt werden kann: Sie beschränken sich meist auf eine geringe Fallzahl und die DNA-Analysen beruhen häufig auf der Verwendung von archiviertem Tumormaterial, womit in der Regel nur eine unzureichende Messpräzision erreicht werden kann.

Da Teilergebnisse dieser mit 279 Fällen umfangreichsten Studie bereits an anderer Stelle veröffentlicht wurden [9, 10, 23], soll hier ein kritischer Überblick über die klinische Bedeutung hoch-auflösender DNA-Durchflusszytophotometrie für die Dignitätsdiagnostik bei Tumoren der Speicheldrüsen im Vordergrund stehen.

\section{Patienten und Methoden}

Das Untersuchungskollektiv bestand aus 259 Patienten (129 Frauen, 130 Männer) mit insgesamt 279 Speicheldrüsentumoren, die zwischen 1986 und 2002 am Bundewehrkrankenhaus Ulm operativ versorgt wurden. Das Alter der Patienten betrug 12 bis 85 Jahre mit einem Durchschnitt von 49 Jahren. Die histologische Diagnose erfolgte an Paraffin-eingebetteten Gewebeschnitten nach standardisierter HE-Färbung. Alle Fälle wurden in Übereinstimmung mit den WHO-Richtlinien nachklassifiziert [3]. Tabelle 1 zeigt die Lokalisation der Tumoren, die diagnostische Zuordnung wurde in den Tabellen 2 und 3 zusammengefasst. 
Tabelle 1 Lokalisation der Speicheldrüsentumoren

\begin{tabular}{|l|l|l|}
\hline Patienten & Tumoren & Lokalisation \\
\hline 228 & 246 & Glandula parotis \\
\hline 14 & 14 & Glandula submandibularis \\
\hline 1 & 1 & Glandula sublingualis \\
\hline 16 & 18 & kleine Speicheldrüsen \\
\hline
\end{tabular}

Tabelle 2 Diagnosen und DNA-Ploidie bei 229 benignen Speicheldrüsentumoren

\begin{tabular}{|l|l|l|l|}
\hline Diagnose & Anzahl Tumoren & Diploid & Aneuploid \\
\hline Basazelladenom & 7 & 7 & 0 \\
\hline Lipom & 2 & 2 & 0 \\
\hline Lymphangiom & 1 & 1 & 0 \\
\hline Myoepitheliom & 1 & 1 & 0 \\
\hline Neurofibrom & 1 & 1 & 0 \\
\hline Onkozytom & 1 & 1 & 0 \\
\hline Pleomorphes Adenom & 160 & 160 & 0 \\
\hline Pleomorphes Adenom, Rezidiv & 4 & 4 & 0 \\
\hline Zystadenom & 1 & 1 & 0 \\
\hline Zystadenolymphom (Warthin-Tumor) & 51 & 51 & 0 \\
\hline Summe & 229 & 229 & 0 \\
\hline
\end{tabular}


Tabelle 3 Diagnosen und DNA-Ploidie bei 50 malignen Speicheldrüsentumoren

\begin{tabular}{|l|l|l|l|}
\hline Diagnose & Anzahl Tumoren & Diploid & Aneuploid \\
\hline Adenoid-zystisches Karzinom & 18 & 16 & 2 \\
\hline Azinuszellkarzinom & 5 & 4 & 1 \\
\hline Basalzelladenokarzinom & 1 & 1 & 0 \\
\hline Epithelial-myoepitheliales Karzinom & 3 & 2 & 1 \\
\hline Karzinom im pleomorphen Adenom & 5 & 2 & 3 \\
\hline Mukoepidermoidkarzinom & 10 & 8 & 2 \\
\hline NOS-Adenokarzinom & 1 & 1 & 0 \\
\hline Papilläres Zystadenokarzinom & 2 & 2 & 0 \\
\hline Plattenepithelkarzinom & 1 & 1 & 0 \\
\hline Speichelgangkarzinom & 1 & 1 & 0 \\
\hline Speichelgangzyste mit entzündlicher & & & \\
\hline Plattenepithelmetaplasie & 1 & 0 & 1 \\
\hline Undifferenziertes Karzinom & 2 & 0 & 2 \\
\hline Summe & 50 & 38 & 12 \\
\hline
\end{tabular}

Für die DNA-durchflusszytophotometrischen Untersuchungen wurden ausschließlich frische, unfixierte Tumorproben eingesetzt. Das Gewebe wurde durch gegenläufige Schneidebewegungen zweier chirurgischer Skalpelle sorgfältig bis zu einer breiartigen Konsistenz zerkleinert. Zur Isolierung der Zellkerne wurde das Homogenisat in eine saure Lösung (pH-Wert 1,5) aus $0,5 \mathrm{~g}$ Pepsin in $0,05 \mathrm{NHCl}$ gebracht und für fünf Minuten unter

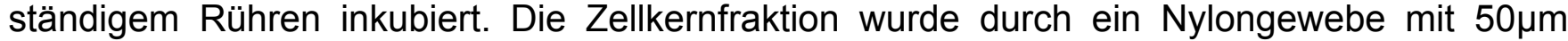
Maschenweite getrennt, dann zentrifugiert $(500 \mathrm{~g}, 10 \mathrm{~min})$, in $70 \%$ Ethanol fixiert und bis zur Messung bei $-20^{\circ} \mathrm{C}$ aufbewahrt.

Für die durchflusszytophotometrische Untersuchung wurden die fixierten Zellkerne sedimentiert (500g, 10min). Zur Vermeidung von Klumpenbildung wurde das Pellet in $0,2 \mathrm{ml}$ Pepsinlösung suspendiert und mehrfach mit einer Pasteurpipette aspiriert. Nach drei Minuten wurden 4,8ml DNA-spezifische Färblösung hinzugefügt. Diese bestand für Messungen, die vor 1991 durchgeführt wurden, aus 10mg Ethidiumbromid (Serva, Heidelberg, Deutschland), 4mg Mithramycin (Serva) und 500mg Magnesiumchlorid $\left(\mathrm{MgCl}_{2}\right)$ gelöst in 1l Tris-Puffer ( $\mathrm{pH}-$ Wert 7,5). Ab 1992 wurde die Färbung auf eine Lösung bestehend aus 0,2mg 4',6-diamidino-2phenylindol (DAPI) und 11,8g Zitronensäure-Trinatrium-Dihydrat (Serva) gelöst in $100 \mathrm{ml}$ Wasser $(\mathrm{pH}-$ Wert 8,0$)$ umgestellt. Die Inkubationsdauer betrug mindestens 30 Minuten bei Raumtemperatur.

Für die DNA-Analysen kamen mit HBO-100 Quecksilber-Hochdrucklampe ausgestatte PAS II oder PAS III Durchflusszytophotometer zur Anwendung (Partec, Münster, Deutschland). Die 
Geräte wurden regelmäßig mit Zellkernen tonsillärer Lymphozyten justiert. Es wurden in der Regel 10.000 Zellen gemessen und als Histogramm gespeichert.

DNA-Histogramme mit einem einzigen Hauptgipfel wurden als diploid eingestuft, ein zusätzlicher Gipfel mit abweichendem Modalwert wurde als aneuploide Tumorzellpopulation gewertet (Abb. 1). Die S-Phasen-Fraktion wurde mit dem Computerprogramm DPAC (Partec) berechnet. Bei aneuploiden Tumoren wurden die SPF-Werte ausschließlich auf den aneuploiden Zellzyklus bezogen. Der Variationskoeffizient, der ein Maß für die Sensitivität der Messung darstellt, schwankte zwischen $0,8 \%$ und $7,0 \%$ mit einem Mittelwert von 2,1\% $( \pm 1,0)$.
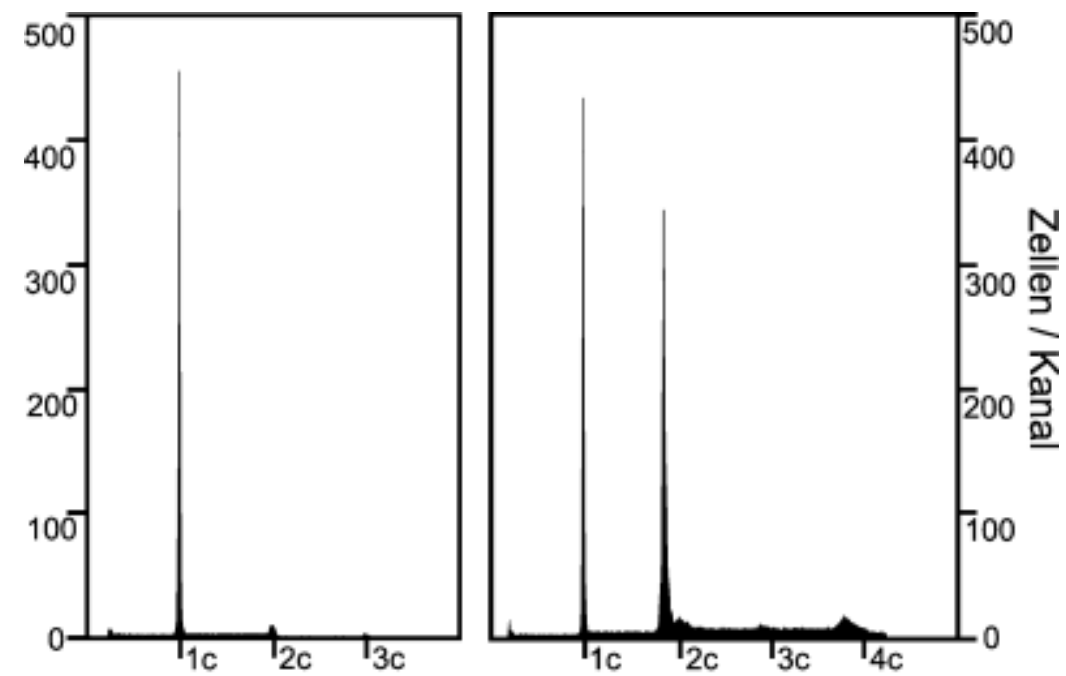

a Relativer DNA-Gehalt b Relativer DNA-Gehalt

Abb. 1 Das DNA Histogramm eines diploiden Tumors (a) weist einen prominenten Gipfel auf, der die Zellen in der G0/G1-Phase des Zellzyklus enthält. Der kleinere Gipfel mit genau doppeltem DNA-Gehalt spiegelt die Zellen in der G2-Phase und Mitose wider. S-Phase-Zellen mit intermediärem DNA-Gehalt können durch Integration der Zellzyklusphasen-Anteile quantitativ erfasst werden. Im Histogramm eines aneuploiden Tumors (b) fällt neben einem diploiden Gipfel, der die immer vorhandenen, nicht-neoplastischen Begleitzellen charakterisiert, ein zweiter Hauptgipfel mit einem relativen DNA-Gehalt von 1,8 auf. Dieser markiert die G0/G1-Zellen der malignen Tumorzellpopulation. Die korrespondierenden G2/M-Zellen bilden einen kleineren Gipfel mit einem relativen DNAGehalt von 3,6. Zellen in der Replikationsphase können durch Integration der aneuploiden Zellpopulation bestimmt werden

\section{Ergebnisse}

Alle 229 als benigne klassifizierten Neoplasien der Speicheldrüsen waren DNA-diploid (Tabelle 2), während 12 von 50 malignen Tumoren (24\%) Ziellinien mit DNAGehaltsaberrationen aufwiesen (Tabelle 3). Ein Zusammenhang zwischen der DNA-Ploidie, Tumorgröße oder histopathologischem Grading wurde nicht festgestellt. Sechs Malignome mit zervikalen Metastasen waren DNA-diploid. Zwei der fünf Karzinome im pleomorphen Adenom wurden unmittelbar aufgrund histologischer Befunde als Karzinom im pleomorphen Adenom diagnostiziert. Beide Tumoren waren DNA-diploid. Drei Karzinome im pleomorphen Adenom erfüllten im Rahmen der Routinediagnostik zunächst das morphologische Erscheinungsbild eines pleomorphen Adenoms. Ein aneuploider Befund gab Anlass für eine intensivere 
Begutachtung. In konsekutiven Schnitten wurden Zellnester erkennbar, welche die endgültige Typisierung als nicht invasives Karzinom im pleomorphen Adenom notwendig machten.

Die Berechnung der S-Phasen-Fraktion (SPF) bei aneuploiden Zellpopulationen ergab Werte zwischen $5,0 \%$ und $40,2 \%$ mit einem Mittelwert von 19,7\% $( \pm 11,7 \%)$. Bei diploiden Malignomen wurden SPF-Werte zwischen $0,9 \%$ und $11 \%$ mit einem Mittelwert von 3,9\% $( \pm 2,6)$ festgestellt. Für die beiden häufigsten benignen Speicheldrüsentumoren wurden bei den pleomorphen Adenomen SPF-Werte von $0,5 \%$ bis $7,9 \%$ mit einem Mittelwert von $2,7 \%( \pm 1,6)$ und bei Zystadenolymphomen (Warthin-Tumoren) von 1,0\% bis 8,9\% mit einem Mittelwert von $3,7 \%( \pm 2,2)$ berechnet. Der Unterschied zwischen beiden Entitäten erwies sich als signifikant $(p<0.01$; U-Test nach Mann und Whitney). Bei den selteneren benignen Tumoren wurden SPF-Werte zwischen $0.9 \%$ und $4.7 \%$ mit einem Durchschnitt von $2.9 \%( \pm 1.5)$ ermittelt. Auf eine statistische Analyse wurde aufgrund der geringen Fallzahl verzichtet. Im Vergleich zu den diploiden Malignomen erwies sich der Unterschied der SPF-Werte für die pleomorphen Adenome als signifikant $(p<0.05)$, wobei sich $4 \%$ als der Wert mit der höchsten Trennschärfe herausstellte, nicht jedoch für die Gruppe der Zystadenolymphome (Warthin-Tumoren) (Abb. 2).

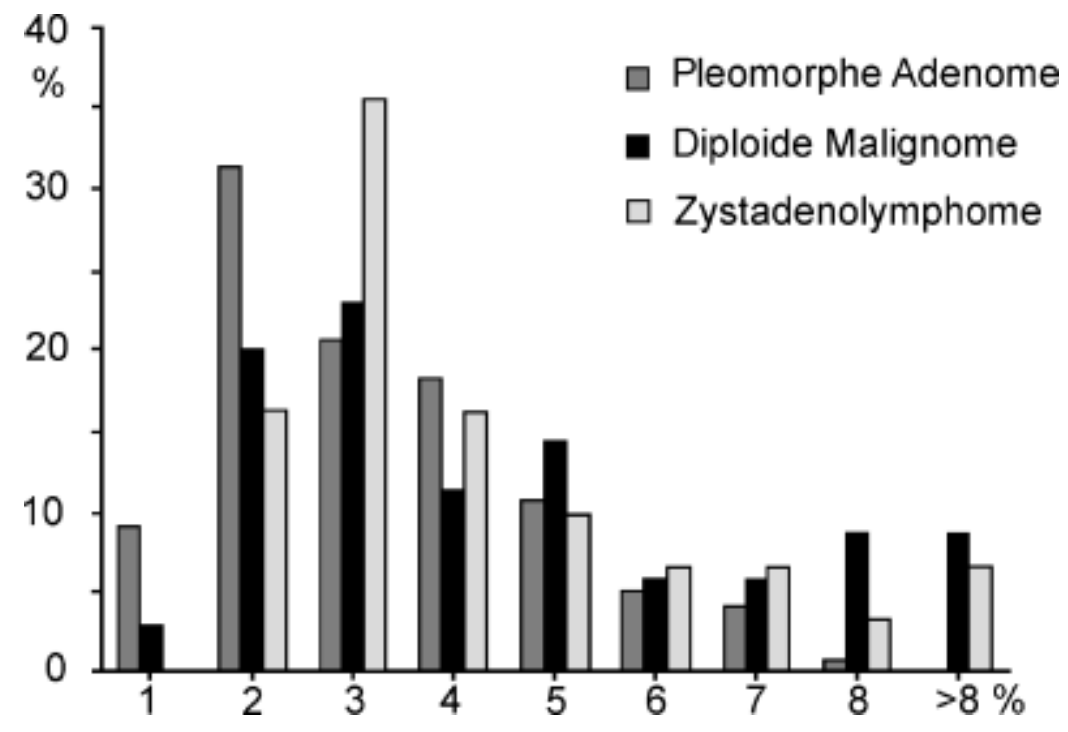

Abb. 2 Verteilung der SPF-Werte für pleomorphe Adenome, Zystadenolymphome (Warthin-Tumoren) und diploide Speicheldrüsentumoren

\section{Diskussion}

Methodische Grundlage der DNA-Durchflusszytophotometrie (FCM) ist die photometrische Quantifizierung des zellulären DNA-Gehaltes mithilfe stöchiometrisch bindender, Nukleinsäure-spezifischer Fluorochrome. Mit dieser Technik können chromosomale Modifikationen dargestellt werden, soweit diese zu einer Veränderung der zellulären DNAMenge führen. Der Umfang karyotypischer Aberrationen spiegelt sich im Grad der DNAGehaltsabweichung vom normalen diploiden Wert somatischer Zellen wider. Der Nachweis klonaler DNA-Aneuploidie beruht im Wesentlichen auf Abweichungen von der regulären 
Chromosomenzahl, während isolierte Veränderungen der chromosomalen Struktur, wie sie vielfach auch für benigne Tumoren beschrieben werden, in der Regel unerkannt bleiben [24, 25]. Solche Strukturanomalien können zwar in einem gewissen Umfang durch vergleichende genomische Hybridisierung (CGH) oder durch Fluorezenz in situ Hybridisierung mit Chromosomen-spezifischen DNA-Proben sichtbar gemacht werden [27], für den Nachweis reiner Chromosomenzahl-Aberrationen sind diese zudem arbeitsaufwendigen Methoden aber nur eingeschränkt geeignet. Tatsächlich gehen aber besonders bei soliden Malignomen numerische Chromosomen-Anomalien häufig mit simultanen Veränderungen der chromosomalen Architektur einher [27].

Diese als Aneuploidie bezeichnete karyotypische Reorganisation stellt ein eindeutiges, wenn auch nicht obligates Malignitätskriterium dar [8]. Da auch geringfügige Veränderungen des klonalen DNA-Gehaltes ein eindeutiges Zeichen maligner Transformation darstellen [18, 24], hat die Sensitivität der Messtechnik für den Nachweis auch einzelner fehlender oder überzähliger Chromosomen eine elementare Bedeutung für die Qualität der Dignitätsdiagnostik. Durch die Entwicklung hochauflösender DNA-Durchflusszytophotometrie, die im Wesentlichen auf der Verwendung des außerordentlich sensitiven DNA-spezifischen Farbstoffes DAPI beruht, konnte dieser Anspruch weitgehend erfüllt werden, auch wenn balancierte numerische Chromosomenanomalien häufig unerkannt bleiben [23].

Offensichtlich aufgrund der geringen Häufigkeit von Speicheldrüsentumoren wurden die bislang durchgeführten DNA-Analysen vielfach an Paraffin-eingebettetem Material durchgeführt $[1,2,5,7,14,16,28,33,40,45,46]$. Die Sensitivität für den Nachweis aneuploider Zellpopulationen ist bei dieser Technik jedoch stark eingeschränkt, für eine Zellzyklusanalyse ist sie kaum geeignet [29]. Nur wenige Studien beruhen auf Untersuchungen an frischen Gewebeproben [38, 43].

Alle der vorliegenden Untersuchung zugrunde liegenden DNA-Analysen wurden ausschließlich an unfixiertem Tumormaterial durchgeführt. Der durchschnittliche Variationskoeffizient von $2,1 \%$ erlaubt den sicheren Nachweis einer DNA-Gehaltsabweichung vom normalen diploiden Wert um ungefähr $4 \%$. Aneusomien des Chromosoms 5, wie sie bei zytogenetischen Untersuchungen an Zellkulturen von Zystadenolymphomen (Warthin-Tumoren) auffielen [32], müssten daher mithilfe hoch-auflösender FCM auch in vivo nachweisbar sein [23]. Ein ausnahmslos diploider DNA-Gehalt bei insgesamt 51 Fällen kann solche numerischen Chromosomenanomalien bei Zystadenolymphomen (Warthin-Tumoren) in vivo nicht bestätigen.

Wie auch bei allen anderen benignen Speicheldrüsentumoren des Untersuchungskollektives, so wurden auch bei der zahlenmäßig größten Gruppe der pleomorphen Adenome ausschließlich DNA-diploide Fälle festgestellt. Dies steht im Einklang mit anderen durchflusszytophotometrischen Untersuchungen [38, 40]. Allerdings berichten einige Studien von gelegentlichem Auftreten aneuploider pleomorpher Adenome [1, 6, 7], so dass die Entwicklung aneuploider Karyotypen bei diesen Tumoren nicht grundsätzlich ausgeschlossen werden kann. Alle vier Rezidive pleomorpher Adenome dieser Studie waren DNA-diploid. Allerdings wurde in einigen Fällen Aneuploidie bei rezidivierten pleomorphen Adenomen dokumentiert $[1,33,39]$. Umfassende Untersuchungen an Plattenepithelkarzinomen der 
Mundhöhle haben nicht nur gezeigt, dass Patienten mit aneuploiden Tumoren ein gegenüber diploiden Fällen dramatisch erhöhtes Risiko einer lokalen und regionalen Rezidivierung tragen [18-20,24], sondern auch dass die aneuploiden Zellen selbst Träger des invasiven und metastasierenden Wachstums sind $[21,26]$. Ob ein solcher Zusammenhang auch für pleomorphe Adenome zutrifft, und gegebenenfalls auch für andere benigne Speicheldrüsentumoren, ist aufgrund der spärlichen Hinweise kaum abzuschätzen. Allerdings würde sich diese Annahme mit der Erfahrung decken, dass Aneuploidie bei als benigne eingestuften Neoplasien zumindest als Hinweis auf ein aggressiveres Wachstumspotential gewertet werden muss [13].

Es sollte noch eine weitere Möglichkeit für das Auftreten aneuploider Zellpopulationen bei pleomeorphen Adenomen in Betracht gezogen werden, nämlich ein unerkanntes Karzinom im pleomorphen Adenom. Aneuploidie ist ein weitverbreitetes Kennzeichen dieser histologisch und zytologisch vielgestaltigen Malignome wie sowohl aus zytogenetischen Untersuchungen [36, 37] als auch aus zytometrischen DNA-Analysen hervorgeht $[4,33,34$, 38]. Tatsächlich wurde bei drei Karzinomen im pleomorphen Adenom der vorliegenden Untersuchung eine eindeutige Diagnose erst nach einer durch einen aneuploiden Befund veranlassten intensiven Begutachtung konsekutiver Schnittebenen gestellt. Auch der histologische Befund einer Speichelgangzyste mit entzündlicher Plattenepithelmetaplasie mit zweifelhafter Dignität sollte aufgrund des Vorliegens einer aneuploidien Zellpopulation als maligne eingestuft werden. Diese Befunde zeigen, dass die DNA-Ploidie-Analyse durchaus zur Bewertung der Dignität von Speicheldrüsentumoren beitragen kann.

Obwohl Aneuploidie ausschließlich bei malignen Tumoren der Speicheldrüsen festgestellt wurden, ist der Anteil dieser Fälle mit $24 \%$ deutlich geringer als bei anderen soliden Malignomen [35]. Dieser Befund wird durch nahezu alle DNA-durchflusszytophotometrischen Untersuchungen bestätigt $[2,5,12,14,16,17,40]$, auch wenn einige Untersuchungen von einem höheren Anteil maligner, aneuploider Speicheldrüsentumoren berichten [11, 30, 46].

Da sich die karyotypische Progression maligner Speicheldrüsentumoren offensichtlich von denen anderer solider Tumoren unterscheidet, stell sich das Problem einer alternativen zytometrischen Differenzierung diploider maligner Formen von benignen Neoplasien der Speicheldrüsen. Wir haben daher untersucht, ob der zweite DNAdurchflusszytophotometrische Parameter, die S-Phasenfraktion (SPF), eine solche Möglichkeit bietet. Die SPF charakterisiert proliferierende Zellen aufgrund ihres replikationsspezifischen DNA-Gehaltes (vgl. Abb. 1). Allerdings stellt die SPF, anders als die DNA-Ploidie, kein diskretes Merkmal dar sondern einen variablen Funktionsparameter. Zusätzliche Einschränkungen bereitet der Umstand, dass sich die SPF bei diploiden Tumoren immer aus Anteilen neoplastischer und nicht-neoplastischer Zellpopulationen eines Gewebes zusammensetzt, deren Verteilung jedoch unbekannt bleibt.

Bei aneuploiden Speicheldrüsentumoren, bei denen eine Zellzyklusanalyse für maligne Zellpopulationen ohne Einbeziehung normaler diploider Zellen durchgeführt werden konnte, wurden SPF-Werte von $5,0 \%$ bis $40,2 \%$ bestimmt. Für die diploiden malignen Fälle wurden SPF-Werte zwischen $0,9 \%$ und $11,0 \%$ berechnet. Diese Werte sind aus oben genannten Gründen nicht vergleichbar, zeigen aber erwartungsgemäß ein hohes Proliferationspotenzial 
neoplastischer Zellpopulationen. Vergleicht man die SPF diploider Malignome mit denen pleomeorpher Adenome mit Werten zwischen 0,5\% und 7,9\%, so ergibt sich ein signifikanter Unterschied zwischen beiden Gruppen, wobei ein SPF-Wert von 4\% die höchste statistische Trennschärfe erreicht. Allerdings ist ein solcher Unterschied für die zweitgrößte benigne Gruppe, den Zystadenolymphomen (Warthin-Tumoren), nicht nachweisbar.

Zusammenfassend muss der diagnostische Wert der DNA-Durchflusszytophotometrie bei Speicheldrüsentumoren kritisch betrachtet werden. Der Nachweis einer aneuploiden Zellpopulation ist auch bei dieser Tumorgruppe ein sicheres Malignitätskriterium. Obwohl ein erhöhter SPF-Wert bei diploidem Histogramm als unterstützender Hinweis für eine maligne Erkrankung gewertet werden kann, ist die Zuverlässigkeit dieses Parameters für eine Identifizierung diploider Malignome unzureichend. Die Ergebnisse dieser Studie könnten allerdings als Grundlage für die Verbesserung der prätherapeutischen Diagnostik und die Weiterentwicklung zytometrischer Diagnosemethoden von Nutzen sein, wie kürzlich durch DNA-Analysen an Feinnadel-Aspriationsbiopsien an Speicheldrüsentumoren gezeigt werden konnte [15].

Anmerkung Weitere Informationen zur Durchflusszytophotometrie und DNA-Ploidie bei KopfHals-Tumoren unter http://www. aneuploidy.info.

\section{Literatur}

1. Atula T, Grenman R, Laippala P, Klemi PJ (1995) Aneuploidy in salivary gland adenomas. Eur Arch Otorhinolaryngol 252:395-400

2. Bang G, Donath K, Thoresen S, Clausen OP (1994) DNA flow cytometry of reclassified subtypes of malignant salivary gland tumors. J Oral Pathol Med 23:291-297

3. Barnes L, Eveson JW, Reichart P, Sidransky D (2005) World Health Organization classification of tumours. Pathology and genetics of head and neck tumours. Salivary glands. IARC, Lyon, pp 209-281

4. Brandwein M, Huvos AG, Dardick I, Thomas MJ, Theise ND (1996) Noninvasive and minimally invasive carcinoma ex mixed tumor: a clinicopathologic and ploidy study of 12 patients with major salivary tumors of low (or no?) malignant potential. Oral Surg Oral Med Oral Pathol Oral Radiol Endod 81:655-664

5. Cho KJ, el-Naggar AK, Ordonez NG, Luna MA, Austin J, Batsakis JG (1995) Epithelialmyoepithelial carcinoma of salivary glands. A clinicopathologic, DNA flow cytometric, and immunohistochemical study of Ki-67 and HER-2/neu oncogene. Am J Clin Pathol 103:432437

6. Daniele E, Tralongo V, Morello V, Nagar C, Russo A, Bazan V, Dardanoni G, Ciotta S, Nuara R, Tomasino RM (1996) Pleomorphic adenoma and adenoid-cystic carcinoma of salivary glands: immunohistochemical assessment of proliferative activity in comparison with flow-cytometric study. Cell Prolif 29:153-162

7. Desinan L, Scott CA, Pizzolitto S, Avellini C, Bardus P, Rimondi G, Rizzi V, Beltrami CA (1996) DNA flow cytometry and glial fibrillary acidic protein reactivity in pleomorphic adenomas of the salivary glands. Anal Quant Cytol Histol 18:355-360 
8. Dey P (2004) Aneuploidy and malignancy: an unsolved equation. J Clin Pathol 57:12451249

9. Driemel O, Maier H, Kraft K, Haase S, Hemmer J (2005) Flow cytometric DNA ploidy in salivary gland tumours. Oncol Rep 13:161-165

10. Driemel O, Kraft K, Hemmer J (2006) Flow cytometric S-phase fraction contributes to diagnosis of diploid malignant salivary gland tumours. Int J Oral Maxillofac Surg 35:947950

11. Eibling DE, Johnson JT, McCoy JP Jr., Barnes EL, Syms CA, Wagner RL, Campbell J (1991) Flow cytometric evaluation of adenoid cystic carcinoma: correlation with histologic subtype and survival. Am J Surg 162:367-372

12. Franzen G, Nordgard S, Boysen M, Larsen PL, Halvorsen TB, Clausen OP (1995) DNA content in adenoid cystic carcinomas. Head Neck 17:49-55

13. Friedlander ML, Hedley DW, Taylor IW (1984) Clinical and biological significance of aneuploidy in human tumours. J Clin Pathol 37:961-974

14. Gemryd P, Lundquist PG, Tytor M, Hellquist HB, Nordenskjold B (1997) Prognostic significance of DNA ploidy in mucoepidermoid carcinoma. Eur Arch Otorhinolaryngol 254:180-185

15. Gerstner AO, Muller AK, Machlitt J, Tarnok A, Tannapfel A, Weber A, Bootz F (2003) Slidebased cytometry for predicting malignancy in solid salivary gland tumors by fine needle aspirate biopsies. Cytometry 53B:20-25

16. Greiner TC, Robinson RA, Maves MD (1989) Adenoid cystic carcinoma. A clinicopathologic study with flow cytometric analysis. Am J Clin Pathol 92:711-720

17. Hamper K, Mausch HE, Caselitz J, Arps H, Berger J, Askensten U, Auer G, Seifert G (1990) Acinic cell carcinoma of the salivary glands: the prognostic relevance of DNA cytophotometry in a retrospective study of long duration (1965-1987). Oral Surg Oral Med Oral Pathol 69:68-75

18. Hemmer J, Schön E (1993) Cytogenetic progression in oral carcinoma: a DNA flow cytometric study on 317 cases. International Journal of Oncology 3:635-640

19. Hemmer J, Kreidler J, van Heerden WFP, Raubenheimer EJ, Schön E (1995) Flow cytometric cellular DNA content and lymph node metastasis in squamous cell carcinoma of the oral cavity. Int $\mathrm{J}$ of Oncol 6:1237-1242

20. Hemmer J, van Heerden WFP, Raubenheimer EJ, Kreidler J, Schön E (1996) Flow cytometric DNA ploidy and recurrence development in squamous cell carcinoma of the oral cavity. Int J Oncol 8:113-116

21. Hemmer J, Thein T, van Heerden WF (1997) The value of DNA flow cytometry in predicting the development of lymph node metastasis and survival in patients with locally recurrent oral squamous cell carcinoma. Cancer 79:2309-2313

22. Hemmer J, Kraft K, Kreidler J (1998a) The significance of DNA flow cytometry in predicting survival and delayed clinical manifestation of occult lymph node metastasis to the untreated neck in patients with oral squamous cell carcinoma. J Craniomaxillofac Surg 26:405-410

23. Hemmer J, van Heerden WF, Polackova J, Kraft K (1998b) High-resolution DNA flow cytometry in papillary cystadenoma lymphomatosum (Warthin's tumour). J Oral Pathol Med 


\section{7:405-406}

24. Hemmer J, Nagel E, Kraft K (1999) DNA aneuploidy by flow cytometry is an independent prognostic factor in squamous cell carcinoma of the oral cavity. Anticancer Res 19:14191422

25. Hemmer J, Hauser C (2000) Chromosomal composition of aneuploid clones with different DNA contents in head and neck squamous cell carcinomas as determined by combined flow cytometry and fluorescence in situ hybridization. Anal Cell Pathol 20:197-203

26. Hemmer J, Kraft K (2001) Stability of aneuploid clones during oral squamous cell carcinoma metastasis. Anticancer Res 21:1459-1464

27. Hemmer J, Kraft K, van Heerden WF (2006) Correlation between DNA ploidy by flow cytometry and chromosome 3 aberration in oral squamous cell carcinoma. Oncol Rep 15:243-246

28. Kelsch RD, Bhuiya T, Fuchs A, Gentile P, Kahn MA, Fantasia JE (1997) Polymorphous low-grade adenocarcinoma: flow cytometric, p53, and PCNA analysis. Oral Surg Oral Med Oral Pathol Oral Radiol Endod 84:391-399

29. Kumar D, Kumar S, Lyon D (1994) Comparison of flow cytometric DNA content analysis of fresh and formalin-fixed paraffin-embedded solid neoplasms. Mod Pathol 7:354-360

30. Lewis JE, McKinney BC, Weiland LH, Ferreiro JA, Olsen KD (1996) Salivary duct carcinoma. Clinicopathologic and immunohistochemical review of 26 cases. Cancer 77:223-230

31. Lindberg JO, Stenling RB, Rutegard JN (1999) DNA aneuploidy as a marker of premalignancy in surveillance of patients with ulcerative colitis. Br J Surg 86:947-950

32. Mark J, Dahlenfors R, Stenman G, Nordquist A (1990) Chromosomal patterns in Warthin's tumor. A second type of human benign salivary gland neoplasm. Cancer Genet Cytogenet 46:35-39

33. Martin AR, Mantravadi J, Kotylo PK, Mullins R, Walker S, Roth LM (1994) Proliferative activity and aneuploidy in pleomorphic adenomas of the salivary glands. Arch Pathol Lab Med 118:252-259

34. Matturri L, Lavezzi AM, Biondo B, Mantovani M (1996) Cell kinetics of pleomorphic adenomas of the parotid gland. Eur J Cancer B Oral Oncol 32B:154-157

35. Merkel DE, McGuire WL (1990) Ploidy, proliferative activity and prognosis. DNA flow cytometry of solid tumors. Cancer 65:1194-1205

36. Morio T, Morimitsu Y, Hisaoka M, Makishima K, Hashimoto H (2002) DNA copy number changes in carcinoma in pleomorphic adenoma of the salivary gland: a comparative genomic hybridization study. Pathol Int 52:501-507

37. el-Naggar AK, Lovell M, Callender DL, Ordonez NG, Killary AM (1998) Concurrent cytogenetic, interphase fluorescence in situ hybridization and DNA flow cytometric analyses of a carcinoma ex-pleomorphic adenoma of parotid gland. Cancer Genet Cytogenet 107:132-136

38. Pinto AE, Fonseca I, Soares J (1999) The clinical relevance of ploidy and S-phase fraction determination in salivary gland tumors: a flow cytometric study of 97 cases. Cancer 85:273-281

39. Safali M, Celasun B, Gunhan O (2002) DNA cytometry in pleomorphic adenomas with 
cytologic atypia. Anal Quant Cytol Histol 24:325-330

40. Saka T, Yamamoto Y, Takahashi H (1991) Comparative cytofluorometric DNA analysis of pleomorphic adenoma and adenoid cystic carcinoma of the salivary glands. Virchows Arch B Cell Pathol Incl Mol Pathol 61:255-261

41. Sen S (2000) Aneuploidy and cancer. Curr Opin Oncol 12:82-88

42. Speight PM, Barrett AW (2002) Salivary gland tumours. Oral Dis 8:229-240

43. Takashima S, Sone S, Horii A, Okamoto S, Yoshida J (1996) Major salivary gland lesions: correlation of MR findings with flow cytometric DNA analysis and prognosis. AJR Am J Roentgenol 167:1297-1304

44. Teodori L, Gohde W, Persiani M, Persiani M, Ferrario F, Tirindelli Danesi D, Scarpignato C, Di Tondo U, Alo P, Capurso L (1998) DNA/protein flow cytometry as a predictive marker of malignancy in dysplasia-free Barrett's esophagus: thirteen-year follow-up study on a cohort of patients. Cytometry 34:257-263

45. Timon Cl, Dardick I, Panzarella T, Patterson B, Thomas MJ, Ellis GL, Gullane PJ (1994) Acinic cell carcinoma of salivary glands. Prognostic relevance of DNA flow cytometry and nucleolar organizer regions. Arch Otolaryngol Head Neck Surg 120:727-733

46. Tytor M, Gemryd P, Wingren S, Grenko RT, Lundgren J, Lundquist PG, Nordenskjold B (1993) Heterogeneity of salivary gland tumors studied by flow cytometry. Head Neck 15:514-521 